\title{
Functional Inflammatory Genotypes in Ischemic Stroke: Could We Use Them to Predict Age of Onset and Long-Term Outcome?
}

\author{
Stella Marousi, ${ }^{1}$ Anna Antonacopoulou, ${ }^{2}$ Haralambos Kalofonos, ${ }^{2}$ \\ Panagiotis Papathanasopoulos, ${ }^{1}$ Marina Karakantza, ${ }^{3}$ and John Ellul ${ }^{1}$ \\ ${ }^{1}$ Department of Neurology, University Hospital of Patras, 26500 Rion, Patras, Greece \\ ${ }^{2}$ Clinical Oncology Laboratory, University Hospital of Patras, 26500 Rion, Patras, Greece \\ ${ }^{3}$ Department of Heamatology, University Hospital of Patras, 26500 Rion, Patras, Greece
}

Correspondence should be addressed to Stella Marousi, smarousi@upatras.gr

Received 30 December 2010; Revised 20 February 2011; Accepted 28 February 2011

Academic Editor: Leonardo Pantoni

Copyright (c) 2011 Stella Marousi et al. This is an open access article distributed under the Creative Commons Attribution License, which permits unrestricted use, distribution, and reproduction in any medium, provided the original work is properly cited.

\begin{abstract}
Functional single-nucleotide polymorphisms (SNPs) of inflammatory cytokines have been previously related to the occurrence of an ischemic stroke (IS). We investigated whether five functional SNPs (i.e., TNF- $\alpha-308 \mathrm{G}>\mathrm{A}$, IL6-174G $>$ C, IL12B 1188A $>$ C, IL4-589C $>$ T, and IL10-1082G>A) might be associated with the age of onset and 6-month outcome of an acute IS. A probe-free real-time PCR methodology was used to genotype 145 consecutively admitted cases with a first-ever IS. Simple Kaplan-Mayer and adjusted Cox regression analyses showed no association between inflammatory genotypes and the age of IS onset. IL6-174G>C, IL12B 1188A>C, IL4-589C > T, and IL10-1082G>A were not found to significantly contribute to the long-term outcome of the disease. However, carriage of the TNF- $\alpha-308$ GG genotype was significantly associated with reduced odds for an adverse outcome. Larger studies are needed to confirm our results.
\end{abstract}

\section{Introduction}

In the last decade, inflammatory mechanisms have been implicated both in the manifestation and evolution of brain ischemia [1]. Immune inflammatory processes may in time predispose to ischemic stroke (IS) through precipitation of atherosclerotic disease [2] and sustainment of atrial fibrillation [3]. Moreover, salvation of the ischemic penumbra and final size of the brain infarct, both of which strongly correlate to clinical outcome after an IS, seem to be massively regulated by networks of poststroke inflammatory responses $[4,5]$. Thus, proinflammatory and anti-inflammatory cytokines are important mediators of the pathophysiological events which precede an acute IS [6] and have been further related to the clinical outcome of the disease (reviewed in [7]).

Among the various proinflammatory cytokines, tumor necrosis factor-alpha (TNF- $\alpha$ ) and interleukin (IL) 6 seem to actively participate in the immune-mediated inflammation of stroke [5,8]. IL12 is an important regulatory cytokine with proinflammatory inclination [9], which is also found upregulated during stroke [10]. IL4 and IL10 are cytokines of the anti-inflammatory arm which counteract the actions of proinflammatory molecules, and have been found suppressed prior to, as well as after a cerebral ischemic event [11-13].

On grounds of genetic regulation, the production of these inflammatory cytokines seems to be significantly influenced by the presence of point mutations located within regulatory gene regions, known as functional singlenucleotide polymorphisms (SNPs) [14]. SNP-association studies of inflammatory cytokines have been previously conducted in cases of IS, under the hypothesis that a genetic predisposition to enhanced (or suppressed) production of T-helper 1 (Th1) or Th2 cytokines could possibly modify the risk of the disease (reviewed in [15]). However, so far, none of these studies has addressed the question of whether such functional genetic variances could predispose to the premature onset of cerebrovascular disease or alter its clinical outcome.

In the present study, we investigated the possible impact of five common SNPs into the age of onset and long-term 
TABLE 1: Allele-specific primers and real-time PCR conditions.

\begin{tabular}{|c|c|c|}
\hline & Allele-specific primers & Real-time PCR conditions \\
\hline \multicolumn{3}{|l|}{ IL4-589C $>\mathrm{T}$} \\
\hline FRW C primer & 5'-GCGGCGCGGCCCACTAAACTTGGGAGAACATTGA & \multirow{4}{*}{$\begin{array}{l}\text { Segment 1-Amplification ( } 40 \text { cycles) } \\
\text { (i) Denaturation at } 95^{\circ} \mathrm{C} \text { for } 30^{\prime \prime}\end{array}$} \\
\hline FRW T primer & 5'-AGTAAACTTGGGAGAACATTGTT-3' & \\
\hline REV primer & 5'-GGCAGAATAACAGGCAGAC-3' & \\
\hline IL10-1082G>A & & \\
\hline $\begin{array}{l}\text { FRW G primer } \\
\text { FRW A primer }\end{array}$ & $\begin{array}{l}5^{\prime}-G C G G C G C G G C G G \text {-TTAGTAAGGCTTCTTTGGGTG - } 3^{\prime} \\
5^{\prime} \text {-TACTACTAAGGCTTCTTTGGGAA - } 3^{\prime}\end{array}$ & (ii) Annealing at $56^{\circ} \mathrm{C}$ for $30^{\prime \prime}$ \\
\hline REV primer & 5'-CTGGATAGGAGGTCCCTTAC-3' & (iii) Extension at $72^{\circ} \mathrm{C}$ for $20^{\prime \prime}$ \\
\hline \multicolumn{3}{|l|}{ TNF- $\alpha-308 \mathrm{G}>\mathrm{A}$} \\
\hline FRW G primer* & 5'-GCGGCGCGGCGG-CTGGCTGAACCCCGTCTC-3' & \multirow{3}{*}{$\begin{array}{l}\text { Segment 2-Qualitative data collection (115 } \\
\text { cycles): }\end{array}$} \\
\hline FRW A primer* & $5^{\prime}$-AGGCTGAACCCCGTG $\bar{C}$ CT-3' & \\
\hline REV primer* & 5'-AAGGAAACAGACCACAGACCTG-3' & \\
\hline \multicolumn{2}{|l|}{ IL6-174G >C } & \multirow{4}{*}{$\begin{array}{l}70-95^{\circ} \mathrm{C} \text { for } 18^{\prime \prime} \\
(\text { Specifically for TNF- } \alpha-308 \mathrm{G}>\mathrm{A} \text { : } \\
\text { annealing at } 61^{\circ} \mathrm{C} \text { for } 25^{\prime \prime} \text { ) }\end{array}$} \\
\hline FRW G primer* & 5'-ATTGTGACGTCCTTTAGCATC-3' & \\
\hline FRW C primer* & $5^{\prime}-\overline{-G C} G G G C G G G C C G$-ATTGTGACGTCCTTTAGGTTG-3' & \\
\hline REV primer* & $5^{\prime}$-ATGACGACCTAAGCTGCAC-3' & \\
\hline \multicolumn{3}{|l|}{ IL12B $1188 \mathrm{~A}>\mathrm{C}$} \\
\hline FRW A primer* & 5'-TTTCAATGAGCATTTAGCAA_CT-3' & \\
\hline FRW C primer* & 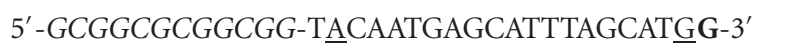 & \\
\hline REV primer* & 5'-TAGGATCACAATGATATCTTTGC-3' & \\
\hline
\end{tabular}

* Primer designed on the complementary sequence. FRW: forward, REV: reverse.

Underlined are sites of introduced mismatches. Bold are the polymorphic alleles. Italic is the intoduced GC-tail.

functional outcome of an acute IS. The studied mutations included three SNPs of proinflammatory cytokines (i.e., TNF- $\alpha-308 \mathrm{G}>\mathrm{A}$, IL6-174G $>$ C, and IL12B $1188 \mathrm{~A}>\mathrm{C}$ ), and two more SNPs concerning the major anti-inflammatory cytokines (i.e., IL4-589C>T, and IL10-1082G>A). These SNPs have been previously studied in various inflammatory diseases (e.g., rheumatic diseases, asthma, and inflammatory bowel disease) producing positive correlations [16-20]. Each of the above polymorphisms has been reported to exert functional properties in in vitro and ex vivo studies, following mitogenic stimulation [16, 21-24]. A more robust evidence for their functionality has been given for TNF- $\alpha-308 \mathrm{G}>\mathrm{A}$ which has been found to affect a transcription-binding site and result in the formation of an altered transcription element [25] and also for IL10-1082G>A which contains a putative ETS-like transcription factor binding site [26].

\section{Methods}

2.1. Study Population. We recruited 145 acute IS patients who presented consecutively at the Emergency Department of the University Hospital of Patras, Patras, Greece, with signs and symptoms of a first-ever cerebrovascular event, and were admitted in the Neurology and General Medicine wards between September 2006 and December 2007. Patients with intracerebral or subarachnoid haemorrhage, transient ischemic attacks (TIAs), chronic inflammatory/rheumatic diseases, comorbid malignancy, and strokes secondary to rare conditions (i.e., traumatic carotid dissection, endocarditis) were excluded from the study. Blood samples and baseline clinical data were collected upon admittance to the Emergency Department. Stroke severity on admission was assessed using the Scandinavian stroke scale. All patients had a brain computerized tomography (CT) on admission, and a second brain imaging (CT or magnetic resonance imaging) during hospitalization. Investigations such as carotid ultrasonography, carotid CT angiography, transesophageal heart ultrasonography, serology and coagulation studies, were performed according to patient-specific clinical criteria. Stroke subtype classification was based on TOAST definitions [27]. As confirmed at the baseline interview, participants were Caucasian in origin, and provided their informed consent at inclusion. The study protocol was approved by the Ethical Committee of the University Hospital of Patras.

2.2. Data and Clinical Assessments. Demographics, past medical history, and conventional stroke risk factors were collected at inclusion. Arterial hypertension was considered present if it was clearly documented $(>140 / 90 \mathrm{mmHg})$ or when treated. Diabetes mellitus was defined as fasting glucose $>126 \mathrm{mg} / \mathrm{dL}$ or current use of antidiabetic medication. Hypercholesterolemia was noted if fasting cholesterol levels were $>220 \mathrm{mg} / \mathrm{dL}$ or when treated. History of angina, myocardial infarction and heart failure were assembled in "ischemic heart disease". Smoking, either active or ceased within the last 6 months, was recorded as current. By vascular death, we considered death in relation to vascular complications (i.e., stroke, myocardial infarction, pulmonary embolism, and deep vein thrombosis). Patients were 
TABLE 2: Baseline characteristics and genotype frequencies of IS patients.

\begin{tabular}{|c|c|}
\hline Characteristic & $\begin{array}{c}\text { IS patients } \\
(n=145)\end{array}$ \\
\hline Age, median (IQR) & $68(58-76)$ \\
\hline Sex, females $(\%)$ & $50(34.5)$ \\
\hline Arterial hypertension (\%) & $104(72)$ \\
\hline Diabetes mellitus (\%) & $40(28)$ \\
\hline Hypercholesterolemia (\%) & $102(70)$ \\
\hline Atrial fibrillation (\%) & $31(21)$ \\
\hline Ischemic heart disease (\%) & $37(26)$ \\
\hline Current smoking (\%) & $52(36)$ \\
\hline \multicolumn{2}{|c|}{ Stroke severity on admission (\%) } \\
\hline Mild (SSS = 45-58) & $69(48)$ \\
\hline Moderate (SSS = 19-44) & $44(30)$ \\
\hline Severe (SSS = 0-18) & $32(22)$ \\
\hline \multicolumn{2}{|l|}{ TNF- $\alpha-308 \mathrm{G}>\mathrm{A}$} \\
\hline GG (\%) & $115(79)$ \\
\hline GA $(\%)$ & $30(21)$ \\
\hline \multicolumn{2}{|l|}{ IL6-174G >C } \\
\hline GG (\%) & $85(59)$ \\
\hline GC (\%) & $50(34)$ \\
\hline CC $(\%)$ & $10(7)$ \\
\hline \multicolumn{2}{|l|}{ IL12B 1188A $>$ C } \\
\hline AA (\%) & $77(53)$ \\
\hline $\mathrm{AC}(\%)$ & $57(39)$ \\
\hline CC $(\%)$ & $11(8)$ \\
\hline \multicolumn{2}{|l|}{ IL4-589C $>\mathrm{T}$} \\
\hline CC $(\%)$ & $123(84.8)$ \\
\hline CT (\%) & $22(15.2)$ \\
\hline \multicolumn{2}{|l|}{ IL10-1082G $>A$} \\
\hline AA $(\%)$ & $47(32.4)$ \\
\hline GA (\%) & $71(49)$ \\
\hline GG $(\%)$ & $27(18.6)$ \\
\hline
\end{tabular}

IQR: interquartile range, IS: ischemic stroke,

SSS: scandinavian stroke scale.

followed-up for 6 months registering medications, disease relapses, deaths, and functional outcome measured by the Barthel Index (BI), which is a reliable and widely used disability scale [28].

2.3. DNA Extraction and Genotyping Assays. DNA was extracted from whole blood in EDTA, according to standard purification protocols by Qiagen, QIAamp DNA blood Mini Kit, and stored at $-80^{\circ} \mathrm{C}$ until use. For the genotyping, we applied a real-time polymerase chain reaction (RTPCR) technique, which discriminates alleles by detecting differences in the melting temperatures of the products $\left(T_{\mathrm{m}}\right)$. Differences in $T_{\mathrm{m}}$ are produced by the introduction of a GC-tail at the end of one of the two allele-specific primers [29], and measured by SYBR Green fluorescence. For the studied SNPs, the method was used for the first time. Prototypically designed primers and conditions
TABLE 3: Statistics of the disease-free survival analysis.

\begin{tabular}{|c|c|c|c|}
\hline & \multicolumn{3}{|c|}{ Disease-free survival analysis } \\
\hline & Median (SE) & KM log rank & Cox regression* \\
\hline & IVICUIaII (JE) & $P$ & Odds ratio $(95 \% \mathrm{CI})$ \\
\hline \multicolumn{4}{|c|}{ TNF- $\alpha-308 \mathrm{G}>\mathrm{A}$} \\
\hline GA & $65(4.93)$ & \multirow{2}{*}{.70} & Referent \\
\hline GG & $68(1.97)$ & & $0.74(0.49-1.12)$ \\
\hline \multicolumn{4}{|c|}{ IL6-174G >C } \\
\hline GG & $65(2.01)$ & \multirow{2}{*}{.86} & Referent \\
\hline GC/CC & $69(1.66)$ & & $0.70(0.51-1.22)$ \\
\hline \multicolumn{4}{|c|}{ IL12B $1188 \mathrm{~A}>\mathrm{C}$} \\
\hline AA & $67(2.00)$ & \multirow{2}{*}{.62} & Referent \\
\hline $\mathrm{AC} / \mathrm{CC}$ & $70(2.58)$ & & $1.02(0.72-1.44)$ \\
\hline \multicolumn{4}{|c|}{ IL4-589C>T } \\
\hline CC & $67(2.08)$ & \multirow{2}{*}{.91} & Referent \\
\hline CT & $68(4.10)$ & & $1.07(068-1.70)$ \\
\hline \multicolumn{4}{|c|}{ IL10-1082G $>A$} \\
\hline GA/AA & $68(2.09)$ & \multirow{2}{*}{.55} & Referent \\
\hline GG & $65(6.06)$ & & $1.43(0.92-2.22)$ \\
\hline
\end{tabular}

SE: standard error, KM: Kaplan-Mayer, CI: confidence intervals.

* Adjusted for sex, hypertension, diabetes, hyperlipidemia, atrial fibrillation, ischemic heart disease, and smoking.

can be seen in Table 1. Primers were designed using the DNAman version 4.02 software, tested for specificity with BLAST (http://blast.ncbi.nlm.nih.gov/), and synthesized by Metabion International AG, Germany. All samples were processed in duplicates, and genotyping of $10 \%$ random samples for each SNP was confirmed by sequencing (VBC-BIOTECH Service GmbH, Sequencing Unit, Vienna, Austria). Analyses were employed in the Stratagene Mx3000P qPCR machine, using the Brilliant QPCR MasterMix by Stratagene, La Jolla, USA.

2.4. Statistical Analysis. A Kaplan-Mayer disease-free survival analysis was used to test for possible differences in the age of IS onset, depending on the carriage of specific inflammatory genotype. Statistical significance was expressed with $P \log$ ranks. Cox regression analyses were further conducted for each SNP to include possible known confounders (i.e., sex, hypertension, diabetes, atrial fibrillation, hyperlipidemia, smoking) in the overall effect. To assess the potential genetic contribution in the odds of having a bad functional outcome, a binary logistic regression analysis was applied after adjustments for age, sex, vascular risk factors, stroke severity on admission and TOAST categories. Data of the regressions are given as Odds Ratios (OR) with 95\% confidence intervals ( $95 \% \mathrm{CI})$.

\section{Results}

Baseline characteristics and genotypes of the studied polymorphisms in our IS cohort are seen on Table 2. All genotype frequencies were found in accordance to the Hardy-Weinberg 
TABLE 4: Association of inflammatory genotypes with long-term functional outcome of an IS.

\begin{tabular}{|c|c|c|c|c|c|}
\hline \multirow[b]{2}{*}{ 6-month outcome } & \multicolumn{2}{|c|}{ TNF- $\alpha-308 \mathrm{G}>\mathrm{A}$} & \multicolumn{3}{|c|}{ Presence of GG } \\
\hline & AG & GG & $\mathrm{OR}^{*}$ & $95 \% \mathrm{CI}$ & $P$ \\
\hline \multirow[t]{3}{*}{$\begin{array}{l}\text { (BI: } 16-20) \text { good }(\%) \\
(\mathrm{BI}:<16, \text { or death) bad }(\%)\end{array}$} & $\begin{array}{c}15(17) \\
13(25.5)\end{array}$ & $\begin{array}{c}73(83) \\
38(74.5)\end{array}$ & $\begin{array}{c}\text { Referent } \\
0.19\end{array}$ & $0.04-0.86$ & .03 \\
\hline & \multicolumn{2}{|c|}{ IL6-174G $>C$} & \multicolumn{3}{|c|}{ Presence of GC/CC } \\
\hline & GG & GC/CC & $\mathrm{OR}^{*}$ & $95 \%$ CI & $P$ \\
\hline \multirow[t]{3}{*}{$(\mathrm{BI}:<16$, or death) bad $(\%)$} & $\begin{array}{l}54(64.3) \\
34(62.8) \\
\end{array}$ & $\begin{array}{l}30(35.7) \\
21(38.2) \\
\end{array}$ & $\begin{array}{c}\text { Referent } \\
1.14 \\
\end{array}$ & $0.37-3.54$ & .82 \\
\hline & \multicolumn{2}{|c|}{ IL12B 1188A $>C$} & \multicolumn{3}{|c|}{ Presence of AC } \\
\hline & $\mathrm{AA} / \mathrm{CC}$ & $\mathrm{AC}$ & $\mathrm{OR}^{*}$ & $95 \% \mathrm{CI}$ & $P$ \\
\hline \multirow[t]{3}{*}{ (BI: $<16$, or death) bad $(\%)$} & $\begin{array}{l}48(54.5) \\
37(72.5) \\
\end{array}$ & $\begin{array}{l}40(45.5) \\
14(27.5) \\
\end{array}$ & $\begin{array}{c}\text { Referent } \\
0.69\end{array}$ & $0.23-2.09$ & .52 \\
\hline & \multicolumn{2}{|c|}{ IL4-589C $>\mathrm{T}$} & \multicolumn{3}{|c|}{ Presence of CT } \\
\hline & $\mathrm{CC}$ & CT & $\mathrm{OR}^{*}$ & $95 \% \mathrm{CI}$ & $P$ \\
\hline \multirow[t]{3}{*}{$(\mathrm{BI}:<16$, or death) bad $(\%)$} & $\begin{array}{l}71(84.1) \\
43(84.3) \\
\end{array}$ & $\begin{array}{c}14(15.9) \\
8(15.7) \\
\end{array}$ & $\begin{array}{c}\text { Referent } \\
0.67 \\
\end{array}$ & $0.17-2.59$ & .56 \\
\hline & \multicolumn{2}{|c|}{ IL10-1082G $>A$} & \multicolumn{3}{|c|}{ Presence of GG } \\
\hline & GG & AG/AA & $\mathrm{OR}^{*}$ & $95 \%$ CI & $P$ \\
\hline $\begin{array}{l}\text { BI: } 16-20) \text { good }(\%) \\
(\mathrm{BI}:<16, \text { or death) bad }(\%)\end{array}$ & $\begin{array}{l}14(15.9) \\
12(23.5)\end{array}$ & $\begin{array}{l}74(84.1) \\
39(76.5)\end{array}$ & $\begin{array}{l}\text { Referent } \\
2.65\end{array}$ & $0.57-12.40$ & .22 \\
\hline
\end{tabular}

BI: barthel index, OR: odds ratio, CI: confidence intervals.

* ORs adjusted for age, sex, hypertension, diabetes, hyperlipidemia, ischemic heart disease, smoking, stroke severity on admission and TOAST categories.

equilibrium, that is, for TNF- $\alpha-308 \mathrm{G}>\mathrm{A} P=.17$, for IL6$174 \mathrm{G}>\mathrm{C} P=.48$, for IL12B $1188 \mathrm{~A}>\mathrm{C} P=.92$, for IL4$589 \mathrm{C}>\mathrm{T} P=.32$, and for IL10-1082G $>$ A $P=.98$.

Figure 1 shows the Kaplan-Mayer curves for the diseasefree survival analysis depending on genotypes of each studied inflammatory SNP. As seen on the curves, carriers of the ancestral alleles did not significantly differ in the age of stroke onset compared to their homo- and heterozygous mutant counterparts. Estimates of median (standard error) age of IS onset for each inflammatory genotype, as well as the Kaplan-Mayer $P$ log ranks can be seen on Table 3. A Cox regression analysis was further applied to correct the impact of studied genotypes on the age of disease onset, for other important confounders (i.e., sex, hypertension, diabetes, hyperlipidemia, atrial fibrillation, ischemic heart disease, and smoking). ORs with 95\% CI of the adjusted genotype effects confirmed a lack of association between inflammatory SNPs and disease-free survival (Table 3). Interestingly, in all regressions, the most powerful predictors of the premature age of IS onset were smoking and hyperlipidemia (both $P<.001$, data not shown).

Because Kaplan-Mayer curves (Figure 1) involving TNF$\alpha-308 \mathrm{G}>\mathrm{A}$, IL4-589C $>$ T and IL10-1082G $>$ A presented differentiating slopes approximately before the age of 65 , we reapplied the above analysis separately for this younger age-group ( $n=76)$. However, neither this subanalysis could point to significant differences in the age of IS onset between the different inflammatory genotypes. The subsequent Cox regression (OR (95\% CI)) also showed no significant contribution of TNF- $\alpha$-308GG $(0.70(0.37-1.35)$, IL4-589CT (0.53 (0.26-1.07)), or IL10-1082 GG (1.83 (0.973.47)) genotypes, after standard adjustments for sex and vascular risk factors.

Table 4 contains the genotypic distributions across categories of good and bad functional outcome, at 6 months after IS. Bad functional outcome included cases with $\mathrm{BI}<16$, or death. Combinations of genotypes have been made so as to unite groups of "high" versus "low" cytokine producers. The binary logistic regression showed a statistical significant contribution of the TNF- $\alpha-308$ GG ancestral genotype in the reduction of the odds for an adverse 6month outcome $(\mathrm{OR}(95 \% \mathrm{CI})=0.19(0.04-0.86))$, even after important corrections for age, sex, vascular risk factors, stroke severity on admission and TOAST categories. For the rest of the inflammatory SNPs, no similar association could be confirmed. As expected, stroke severity on admission was the most powerful predictor in the models $(P<.001$, data not shown).

\section{Discussion}

In the last decade, experimental as well as clinical studies have recognized both pro and anti-inflammatory cytokines as key mediators of the low-grade aseptic inflammation, which precedes an ischemic cerebrovascular event and follows its pathophysiological course [1]. SNPs that alter the production rate of inflammatory cytokines secondary to a given trigger, have been previously related to incident IS [15]. The present 
TNF- $\alpha-308 \mathrm{G}>\mathrm{A}, \log$ rank $=0.7$

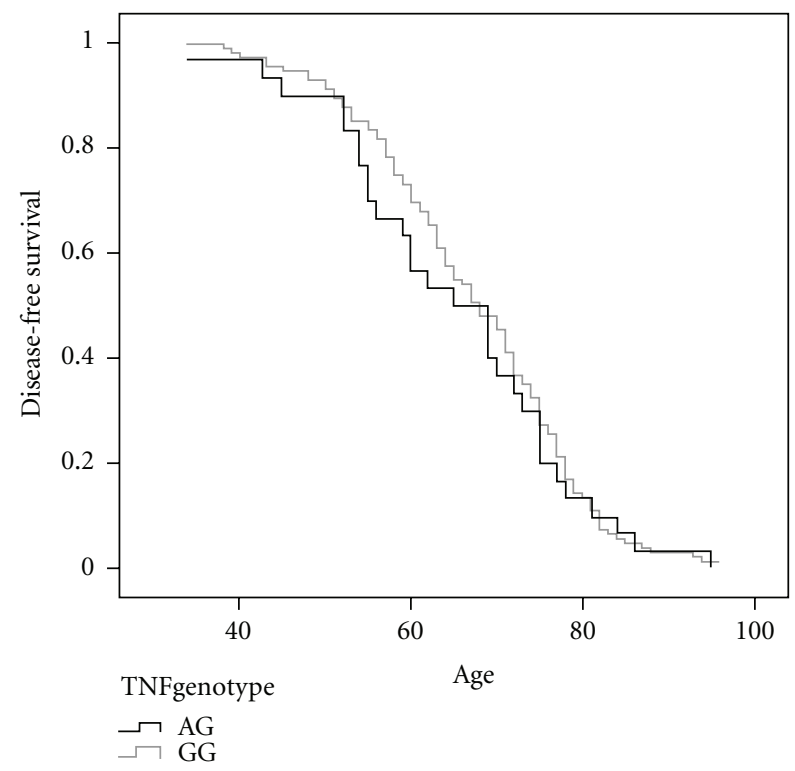

(a)

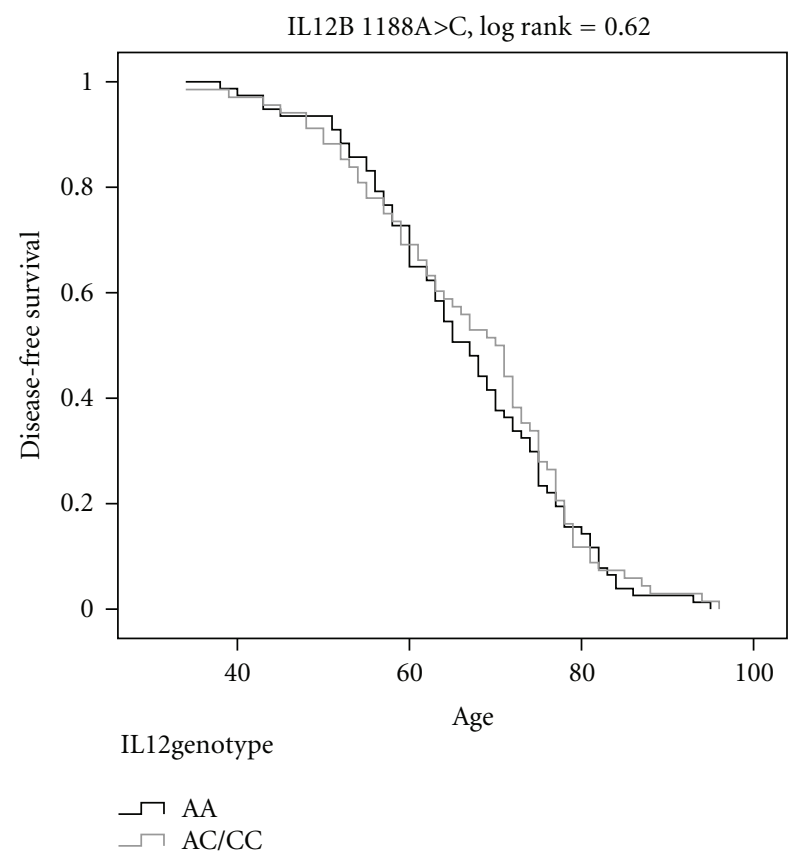

(c)

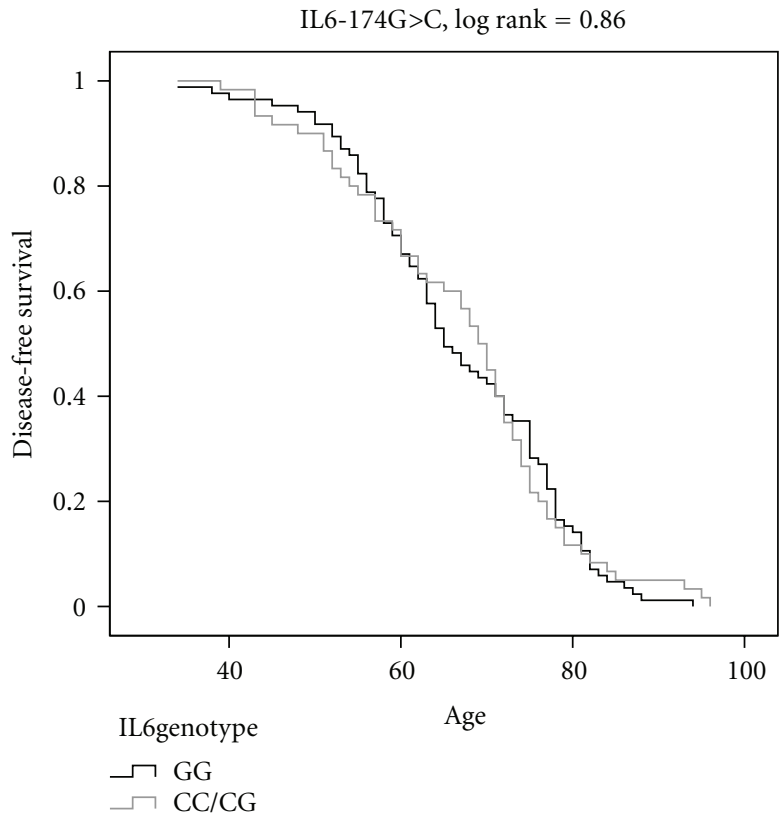

(b)

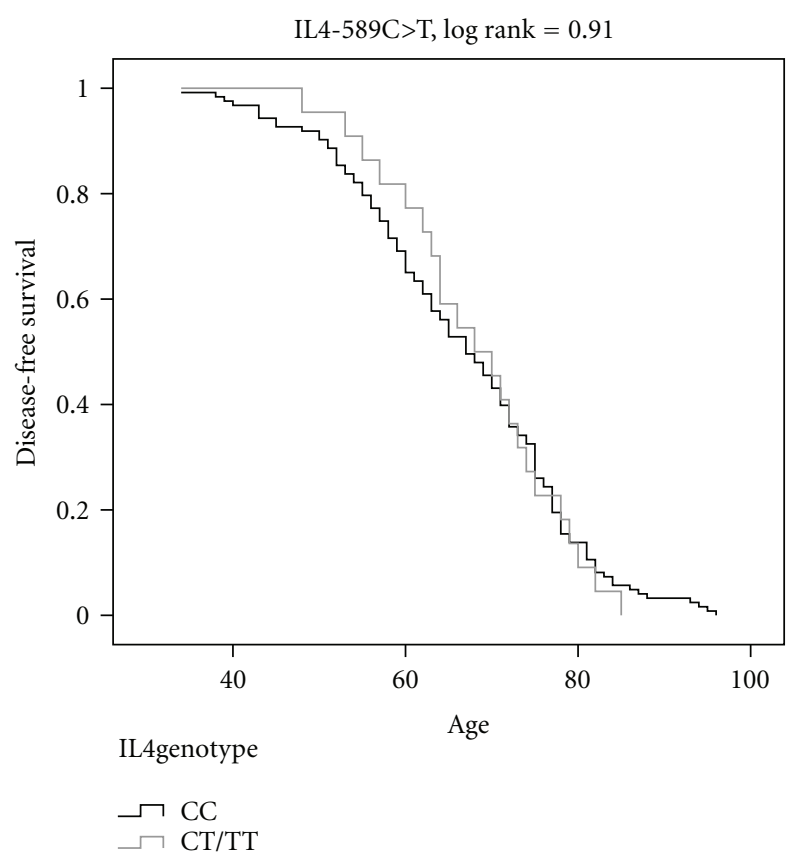

(d)

Figure 1: Kaplan-Mayer curves for the disease-free survival analysis, according to the studied inflammatory genotypes.

study investigated the possible genetic contribution of functional polymorphisms of major inflammatory molecules (i.e., TNF- $\alpha-308 \mathrm{G}>\mathrm{A}$, IL6-174G $>$ C, IL12B $1188 \mathrm{~A}>\mathrm{C}$, IL4$589 \mathrm{C}>\mathrm{T}$ and IL10-1082G $>\mathrm{A}$ ) in the age of onset and 6month outcome of an acute IS. Genotyping was based on a PCR-allele discrimantion technique without oligonucleotide probes [29], which yielded comparable allele frequencies to those previously reported using different methods [30-34].
Despite previous promising data suggesting a significant association of the age of onset of Alzheimer's disease with genotypes of TNF- $\alpha-308 \mathrm{G}>\mathrm{A}$ SNP [35], our results showed that the age of onset of ischemic cerebrovascular disease may not be predicted by inflammatory genotypes. Simple KaplanMayer disease-free survival curves, as well as Cox regressions adjusted for sex and vascular risk factors, confirmed that IS is a complex, multifactorial clinical phenotype, which 
can manifest prematurely or delayed, irrespectively of the genetic inflammatory burden. Furthermore, it is possible that the gross effect of conventional vascular risk factors in the incidence of IS may not be easily overwhelmed. Under this rationale, it would be interesting to know whether the reported genotype frequencies differ from those of a healthy population. This investigation could be the aim of a future case-control association study.

Although genome-wide association studies are reasonably more relevant to detect combinations of genetic traits in relation to a specific clinical phenotype over a large number of patients, they tend to miss important clinical aspects of the disease. This is why, smaller-scale SNP association studies of molecules which have some a priori evidence for a pathogenic role in the disease of interest, may still be useful in implying possible relations with meaningful clinical impact. Under this scope, we set to investigate whether our selected inflammatory functional SNPs could predict the long-term outcome of an IS. After applying adjusted analyses, we found a lack of association between IL6-174G >C, IL12B 1188A $>$ C, IL4-589C $>$ T and IL10-1082G $>$ A and 6-month outcome after stroke. The relatively limited number of IS cases included, may partially explain the above lack of associations. However, we were able to identify a significant contribution of the ancestral TNF- $\alpha-308$ GG genotype in about $80 \%$ reduced odds for having an adverse outcome $(\mathrm{OR}(95 \% \mathrm{CI})=0.19$ $(0.04-0.86))$. This long-term association of TNF- $\alpha-308 \mathrm{G}-$ allele double carriage is in line with its described low TNFsecreting properties [36]. High TNF- $\alpha$ levels at 3 months after an IS, have been previously found to relate with undesired functional dependency [37].

To conclude, our results show that functional genotypes of important inflammatory cytokines cannot either delay or precipitate the age of IS onset, despite the widely accepted role of such molecules in the pathophysiology of the disease. We provide implications for a possible predictive effect of TNF- $\alpha-308$ GG ancestral genotype into better clinical outcome 6 months after stroke. It is possible that the small size of our study may have not allowed for identification of other statistical significant associations. Therefore, further studies in larger IS cohorts, are needed to confirm our results.

\section{Conflict of Interests}

The authors declare that there is no conflict of interests.

\section{Aknowledgment}

S. Marousi was supported by the Research Committee, University of Patras, Patras, Greece (Program K. Karatheodoris B.705).

\section{References}

[1] K. W. Muir, P. Tyrrell, N. Sattar, and E. Warburton, "Inflammation and ischaemic stroke," Current Opinion in Neurology, vol. 20, no. 3, pp. 334-342, 2007.
[2] G. K. Hansson, "Immune mechanisms in atherosclerosis," Arteriosclerosis, Thrombosis, and Vascular Biology, vol. 21, no. 12, pp. 1876-1890, 2001.

[3] M. D. M. Engelmann and J. H. Svendsen, "Inflammation in the genesis and perpetuation of atrial fibrillation," European Heart Journal, vol. 26, no. 20, pp. 2083-2092, 2005.

[4] P. Mergenthaler, U. Dirnagl, and A. Meisel, "Pathophysiology of stroke: lessons from animal models," Metabolic Brain Disease, vol. 19, no. 3-4, pp. 151-167, 2004.

[5] M. Rodríguez-Yáñez and J. Castillo, "Role of inflammatory markers in brain ischemia," Current Opinion in Neurology, vol. 21, no. 3, pp. 353-357, 2008.

[6] M. Cesari, B. W. J. H. Penninx, A. B. Newman et al., "Inflammatory markers and onset of cardiovascular events: results from the Health ABC study," Circulation, vol. 108, no. 19, pp. 2317-2322, 2003.

[7] W. Whiteley, C. Jackson, S. Lewis et al., "Inflammatory markers and poor outcome after stroke: a prospective cohort study and systematic review of interleukin-6," PLoS Medicine, vol. 6, no. 9, Article ID e1000145, 2009.

[8] S. Sotgiu, B. Zanda, B. Marchetti et al., "Inflammatory biomarkers in blood of patients with acute brain ischemia," European Journal of Neurology, vol. 13, no. 5, pp. 505-513, 2006.

[9] G. Trinchieri, "Interleukin-12 and the regulation of innate resistance and adaptive immunity," Nature Reviews Immunology, vol. 3, no. 2, pp. 133-146, 2003.

[10] J. Zaremba and J. Losy, "Interleukin-12 in acute ischemic stroke patients," Folia Neuropathologica, vol. 44, no. 1, pp. 5966, 2006.

[11] E. Van Exel, J. Gussekloo, A. J. M. De Craen, A. Bootsma-van Der Wiel, M. Frölich, and R. G. J. Westendorp, "Inflammation and stroke: the Leiden 85-Plus Study," Stroke, vol. 33, no. 4, pp. 1135-1138, 2002.

[12] F. Perini, M. Morra, M. Alecci, E. Galloni, M. Marchi, and V. Toso, "Temporal profile of serum anti-inflammatory and proinflammatory interleukins in acute ischemic stroke patients," Neurological Sciences, vol. 22, no. 4, pp. 289-296, 2001.

[13] N. Vila, J. Castillo, A. Dávalos, A. Esteve, A. M. Planas, and A. Chamorro, "Levels of anti-inflammatory cytokines and neurological worsening in acute ischemic stroke," Stroke, vol. 34, no. 3, pp. 671-675, 2003.

[14] A. J. Brookes, "The essence of SNPs," Gene, vol. 234, no. 2, pp. 177-186, 1999.

[15] S. Marousi, J. Ellul, and M. Karakantza, "Genetic polymorphisms of type-1 and type-2 inflammatory cytokines in ischaemic stroke," Vascular Disease Prevention, vol. 5, no. 2, pp. 89-103, 2008.

[16] D. Fishman, G. Faulds, R. Jeffey et al., "The effect of novel polymorphisms in the interleukin-6 (IL-6) gene on IL-6 transcription and plasma IL-6 levels, and an association with systemic- onset juvenile chronic arthritis," Journal of Clinical Investigation, vol. 102, no. 7, pp. 1369-1376, 1998.

[17] A. G. Wilson, C. Gordon, F. S. Di Giovine et al., "A genetic association between systemic lupus erythematosus and tumor necrosis factor alpha," European Journal of Immunology, vol. 24, no. 1, pp. 191-195, 1994.

[18] G. Z. Fei, E. Svenungsson, J. Frostegård, and L. Padyukov, "The A-1087IL-10 allele is associated with cardiovascular disease in SLE," Atherosclerosis, vol. 177, no. 2, pp. 409-414, 2004.

[19] G. Morahan, D. Huang, M. Wu et al., "Association of IL12B promoter polymorphism with severity of atopic and nonatopic asthma in children," Lancet, vol. 360, no. 9331, pp. 455459, 2002. 
[20] W. Klein, A. Tromm, T. Griga et al., "Interleukin-4 and interleukin-4 receptor gene polymorphisms in inflammatory bowel diseases," Genes and Immunity, vol. 2, no. 5, pp. 287289, 2001.

[21] A. G. Wilson, F. S. Di Giovine, A. I. F. Blakemore, and G. W. Duff, "Single base polymorphism in the human tumour necrosis factor alpha (TNF $\alpha$ ) gene detectable by NcoI restriction of PCR product," Human Molecular Genetics, vol. 1, no. 5, p. 353, 1992.

[22] D. Seegers, A. Zwiers, W. Strober, A. S. Peña, and G. Bouma, "A TaqI polymorphism in the $3^{\prime}$ UTR of the IL-12 p40 gene correlates with increased IL-12 secretion," Genes and Immunity, vol. 3, no. 7, pp. 419-423, 2002.

[23] L. J. Rosenwasser, D. J. Klemm, J. K. Dresback et al., "Promoter polymorphisms in the chromosome 5 gene cluster in asthma and atopy," Clinical and Experimental Allergy, Supplement, vol. 25, supplement 2, pp. 74-78, 1995.

[24] L. E. N. Rees, N. A. P. Wood, K. M. Gillespie, K. N. Lai, K. Gaston, and P. W. Mathieson, "The interleukin-10_1082 G/A polymorphism: allele frequency in different populations and functional significance," Cellular and Molecular Life Sciences, vol. 59, no. 3, pp. 560-569, 2002.

[25] L. J. Abraham and K. M. Kroeger, "Impact of the -308 TNF promoter polymorphism on the transcriptional regulation of the TNF gene: relevance to disease," Journal of Leukocyte Biology, vol. 66, no. 4, pp. 562-566, 1999.

[26] D. Kube, C. Platzer, A. Von Knethen et al., "Isolation of the human interleukin 10 promoter. Characterization of the promoter activity in Burkitt's lymphoma cell lines," Cytokine, vol. 7, no. 1, pp. 1-7, 1995.

[27] H. P. Adams Jr., B. H. Bendixen, L. J. Kappelle et al., "Classification of subtype of acute ischemic stroke: definitions for use in a multicenter clinical trial. TOAST. Trial of Org 10172 in Acute Stroke Treatment," Stroke, vol. 24, no. 1, pp. 35-41, 1993.

[28] F. I. Mahoney and D. W. Barthel, "Functional evaluation: the barthel index," Maryland State Medical Journal, vol. 14, pp. 6165, 1965.

[29] S. Germer and R. Higuchi, "Single-tube genotyping without oligonucleotide probes," Genome Research, vol. 9, no. 1, pp. 72-78, 1999.

[30] M. Revilla, V. Obach, Á. Cervera, A. Dávalos, J. Castillo, and Á. Chamorro, "A -174G/C polymorphism of the interleukin-6 gene in patients with lacunar infarction," Neuroscience Letters, vol. 324, no. 1, pp. 29-32, 2002.

[31] P. Harcos, J. Laki, P. Kiszel et al., "Decreased frequency of the TNF2 allele of TNF- $\alpha-308$ Promoter polymorphism is associated with lacunar infarction," Cytokine, vol. 33, no. 2, pp. 100-105, 2006.

[32] S. Trompet, D. Pons, A. J. M. De Craen et al., "Genetic variation in the interleukin-10 gene promoter and risk of coronary and cerebrovascular events: the PROSPER study," Annals of the New York Academy of Sciences, vol. 1100, pp. 189198, 2007.

[33] P. A. Costeas, L. Koumas, A. Koumouli, A. KyriakouGiantsiou, and A. Papaloizou, "Cytokine polymorphism frequencies in the Greek Cypriot population," European Journal of Immunogenetics, vol. 30, no. 5, pp. 341-343, 2003.

[34] G. Orozco, M. A. González-Gay, L. Paco et al., "Interleukin 12 (IL12B) and interleukin 12 receptor (IL12RB1) gene polymorphisms in rheumatoid arthritis," Human Immunology, vol. 66, no. 6, pp. 710-714, 2005.

[35] V. Alvarez, I. F. Mata, P. González et al., "Association between the TNF $\alpha$-308 A/G polymorphism and the onset-age of
Alzheimer disease," American Journal of Medical Genetics, vol. 114, no. 5, pp. 574-577, 2002.

[36] N. Braun, U. Michel, B. P. Ernst et al., "Gene polymorphism at position -308 of the tumor-necrosis-factor- $\alpha$ (TNF- $\alpha$ ) in Multiple Sclerosis and its influence on the regulation of TNF$\alpha$ production," Neuroscience Letters, vol. 215, no. 2, pp. 75-78, 1996.

[37] T. J. DeGraba, "The role of inflammation after acute stroke: utility of pursuing anti- adhesion molecule therapy," Neurology, vol. 51, no. 3, pp. S62-S68, 1998. 


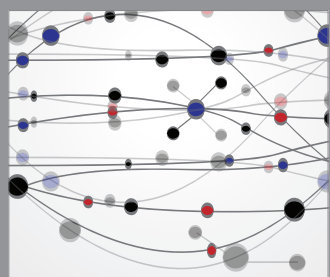

The Scientific World Journal
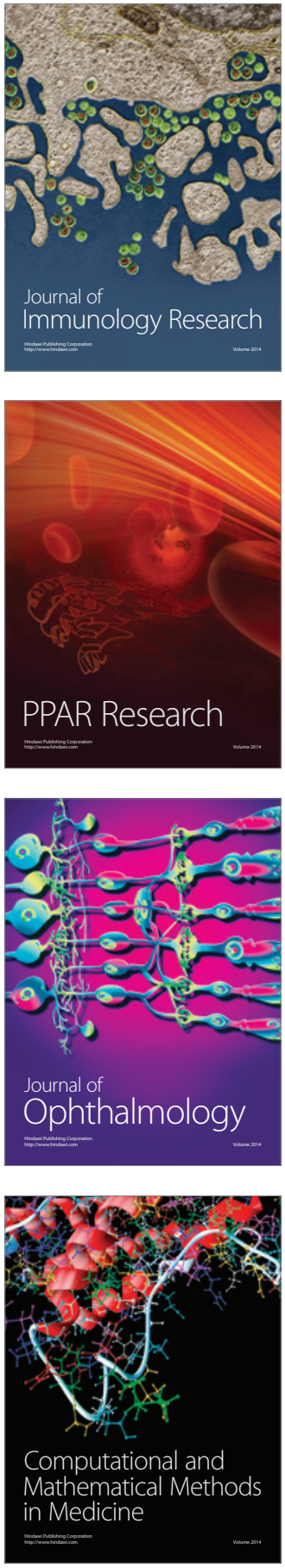

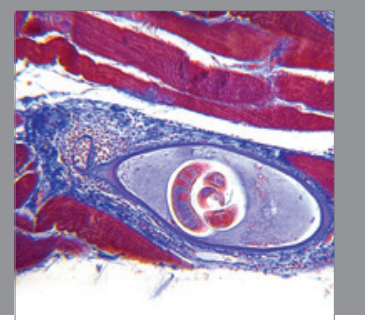

Gastroenterology

Research and Practice
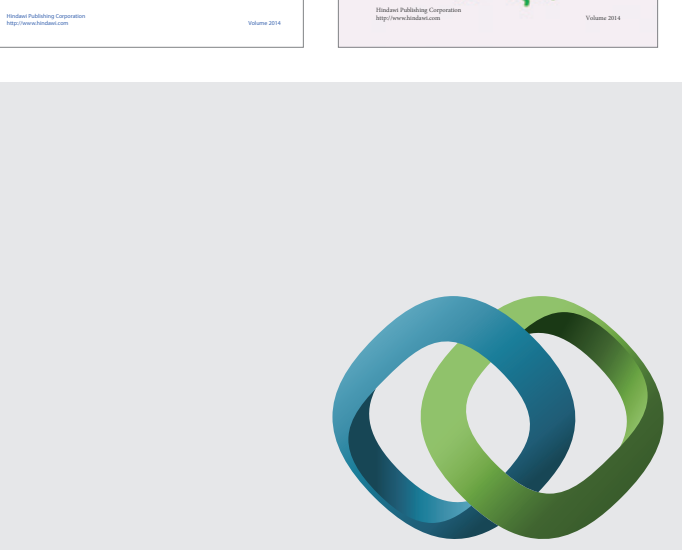

\section{Hindawi}

Submit your manuscripts at

http://www.hindawi.com
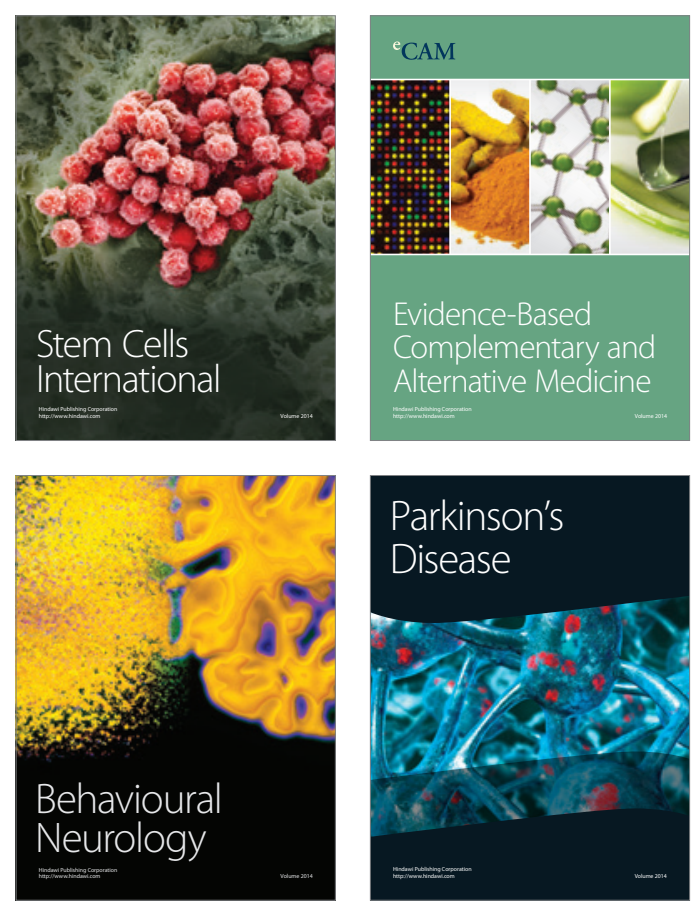

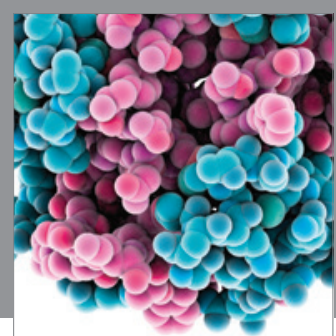

Journal of
Diabetes Research

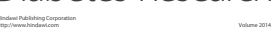

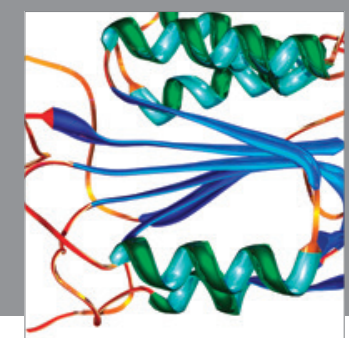

Disease Markers
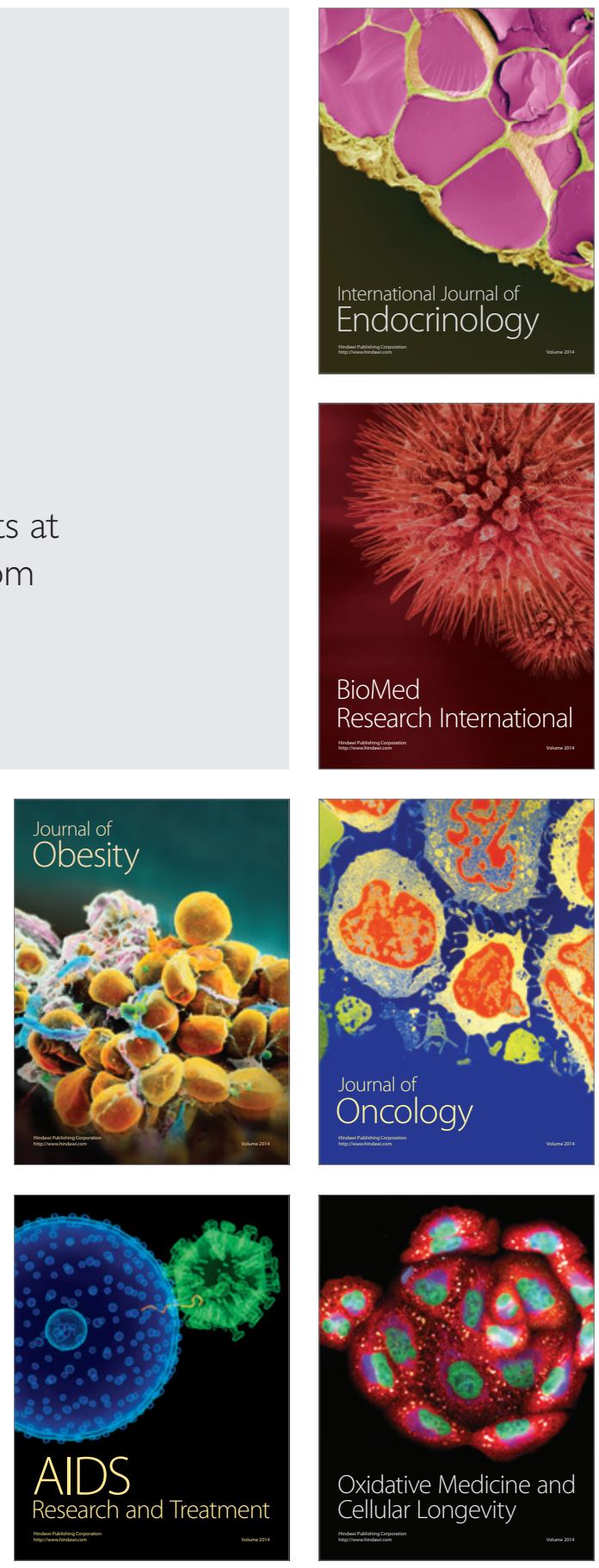